\title{
36 Months
}

National Cancer Institute

\section{Source}

National Cancer Institute. 36 Months. NCI Thesaurus. Code C156844.

A period of time of thirty-six months. 